\title{
Optical-switched proton logic gate: Indocyanine green decorated HSB-W5 MOFs nanosheets
}

\author{
Shuaikang Fan ${ }^{1}$, Shilin Wang ${ }^{1}$, Xiaobin Wang ${ }^{1}$, Xinyi Wan ${ }^{1}$, Zhou Fang ${ }^{1}$, Xiaodong $\mathrm{Pi}^{1}$, Zhizhen Ye $e^{1,2}$ and \\ Xinsheng Peng ${ }^{1,2^{*}}$
}

\begin{abstract}
Proton conduction controlled by light is important for advanced applications in sensors and information processing devices. We herein report a metal-organic framework (MOF) thin film by introducing indocyanine green dye into HSB-W5 (HSB = hydrogenated Schiff base) matrix (ICG@HSB-W5) to construct a series of basic logic gates (NOT, NAND, and NOR) on account of its good photothermal conversion ability and photo-controlled proton conductivity. The proton conductivity of ICG@HSB-W5 thin film is 25 times higher than that of the pristine HSB-W5 thin film and responses efficiently to lasers. In addition, the change of proton conductivity is very large with an ON/OFF ratio above 1000 and enough to be distinguished to build logic gates. In this logic gate system, 808- and $405-\mathrm{nm}$ lasers are used as inputs and the proton conductivity is used as output signal with different thresholds. The electrical output signal is convenient and available to be received and detected. It offers a new route for the design of molecular logic gates based on MOF and facilitates the construction of proton-based logic gate or more complicated logic devices.
\end{abstract}

Keywords: HSB-W5 MOF, proton conductivity, indocyanine green, logic gate, photothermal effect

\section{INTRODUCTION}

Proton conduction and transport in solids are important and useful from battery-related technologies to biological systems. Proton conductive materials are of great interest and expected to be candidates for advanced applications in sensors, supercapacitors, fuel cells, and electronic devices [1-7]. Among many proton-conducting materials, metal-organic frameworks (MOFs) materials display versatile properties, such as tunable porous structures, large specific surface areas, and high crystallinity and structural diversity, and have become one of the most important classes of proton conductive materials [8-12]. These well-known features make MOFs not only nice host materials but also excellent candidates for conducting protons. The intrinsic porosity enables the loading of additional protonic molecules within the pore space, yielding decorated materials with improved proton conductivity. Diverse organic linkers in MOFs materials can be functionalized with various design strategies to allow the introduction of proton conducting media such as water and acidic groups. Proton conduction in MOFs was first reported by Kanda et al. [13] and has captured significant attention over the past few years $[7,14,15]$. Currently, the general proton conduction mechanisms have been extensively studied and many studies focus on achieving high proton conductivity in different operating temperature ranges or switching the proton conductivity simply by external stimuli such as light, heat or electric fields [16-18]. It is worth noting that the efficient and feasible control of proton conduction can work as a signal transducer and provide more opportunities to enable communication between the physical and electronic world $[19,20]$. To explore new dimensions of this interesting research area, using proton conduction in MOFs to construct logic gates is a meaningful attempt with challenge and broadens the path for the development of proton-conductive materials.

The features above-mentioned make MOFs a special molecular system as a signal transducer for the construction of logic devices. Up to now, some studies on logic gates based on different MOFs integrated with other functional materials or groups have been reported by employing chemical molecules or ions, fluorescent or colorimetric signals as input or output [2125 ]. Most of the signals are non-electrical signals and hard to be introduced directly into electrical logic circuits for information detection or processing, which mainly hurdles their further application in electronic devices. The logic gates based on proton-conductive MOFs are promising candidates to avoid this problem and achieve logic operation effectively. For use as a logic gate, the proton conduction system should have at least two stable states, which can be reversibly converted by external stimuli. In most circumstances, light as a convenient, fast and undamaged external trigger has been widely utilized to achieve remote control of different switch due to its high spatiotemporal precision and diversity [26-28].

Recently, a novel neutral MOF, HSB-W1 (HSB = hydrogenated Schiff base), was designed with $\mathrm{Zn}(\mathrm{II})$ as the center and terephthalic acid disodium, and 1,2-bis( $4^{\prime}$-pyridylmethylamino) ethane as the ligands. It is able to be tailored into HSB-W5 with different coordination numbers by treating with water, which has a two-dimensional (2D) twofold interpenetrated layered structure with narrowed channels and good thermal stability. It also exhibits fluorescence emission centered at around $427 \mathrm{~nm}$ upon $310 \mathrm{~nm}$ excitation [29-31]. These features make it a promising host matrix for loading other additives. Nevertheless, this framework is hydrophobic due to the organic linkers, limiting the proton transporting. Indocyanine green (ICG) with two

\footnotetext{
${ }^{1}$ State Key Laboratory of Silicon Materials, School of Materials Science and Engineering, Zhejiang University, Hangzhou 310027, China

${ }^{2}$ Wenzhou Key Laboratory of Novel Optoelectronic and Nanomaterials, Institute of Wenzhou, Zhejiang University, Wenzhou 325006, China

* Corresponding author (email: pengxinsheng@zju.edu.cn)
} 
sulfonate groups in each molecule is an amphiphilic cyanine compound with a special molecular structure [32,33]. Hence, ICG can be introduced into the HSB-W5 pores to improve the water permeability and promote the proton conduction by helping protons "hop" along their conduction pathways or working as a proton carrier in HSB-W5 frameworks through a facile post-synthetic modification approach [34-36]. The strong light absorption of ICG at around $800 \mathrm{~nm}$ has attracted great interest for its excellent photothermal conversion capacity $[37,38]$. This has been widely investigated for photothermal therapy [39-41]. Therefore, it is possible to photoswitch the proton conduction by decorating MOF thin film with ICG molecules to improve proton conductivity and realize photothermal conversion simultaneously. Inspired by our previous work on tuning the proton conduction in MOF thin film via light [28], it is possible to design logic gates by using different lights on the host-guest MOF platform to control different proton conduction states.

As a proof of concept, in this work, we designed opticalresponsive logic gates based on proton conduction by introducing ICG into the HSB-W5 thin film (ICG@HSB-W5) through an infiltration process. In the dark condition, the proton conductivity of ICG@HSB-W5 thin film is approximately $1.91 \times$ $10^{-4} \mathrm{~S} \mathrm{~cm}^{-1}$ at $55^{\circ} \mathrm{C}$ and $95 \%$ relative humidity $(\mathrm{RH})$, which is 25 times higher than that of the pristine HSB-W5 thin film. Upon switching on the lights, the proton conductivity decreases sharply within a few minutes due to the photothermal effect and then restores to its original state reversibly when back to the dark again. The switch factors are 195.4 under 808 -nm laser, 271.0 under $405-\mathrm{nm}$ laser and 1036.3 under the two lasers at the same time, which provides the foundation for proton logic gate construction with lasers as inputs and proton conductivity as output. Utilizing the great change of the proton conductivity, three logic gates (NOT, NAND and NOR) are designed based on the ICG@HSB-W5 thin film according to Boolean logical language. This work offers a possibility for implementation of complicated logic gates or devices and even intelligent molecular machines.

\section{EXPERIMENTAL SECTION}

\section{Materials and reagents}

Zinc nitrate and 2-aminoethanol (AE) were bought from Acros Chemicals. 1,2-Bis(4'-pyridylmethylamino)-ethane was provided by NAFU biology. Terephthalic acid disodium salt was bought from Aladdin. ICG was purchased from Energy Chemical. All reagents were used without further purification. Polycarbonate (PC) membranes with a pore diameter of $200 \mathrm{~nm}$ and an effective diameter of $20 \mathrm{~mm}$ were provided by Whatman and used to filter the mixed solution. Ultrapure water of $18.2 \mathrm{M} \Omega$ was generated using a Millipore direct-Q system in all the experiments.

\section{Synthesis of ICG@HSB-W5 thin film}

The zinc hydroxide nanostrand ( $\mathrm{ZHN}$ ) solution was obtained by adding $4 \times 10^{-3} \mathrm{~mol} \mathrm{~L}^{-1}$ zinc nitrate solution (water:ethanol = $3: 2$ ) into the same volume of $1.6 \times 10^{-3} \mathrm{~mol} \mathrm{~L}^{-1} \mathrm{AE}$ solution (water:ethanol $=3: 2$ ) and then aging for $30 \mathrm{~min}$ at room temperature [42]. The ZHN thin film was obtained by filtering $10 \mathrm{~mL}$ of ZHN solution on a PC membrane. After that, the ZHN thin film on PC membrane was transferred to a piece of glass wetted by ethanol and kept in ethanol for several minutes. Then the membrane was dried for more than $30 \mathrm{~min}$ at room temperature. The HSB-W1 thin film was prepared by immersing the ZHN film into the mixed solution of $25 \times 10^{-6} \mathrm{~mol} \mathrm{~L}^{-1} 1$,2-bis (40-pyridylmethylamino)-ethane solution (water:ethanol $=1: 1$ ) and $22 \times 10^{-6} \mathrm{~mol} \mathrm{~L}^{-1}$ terephthalic acid disodium salt solution $(N, N$-dimethylformamide: water $=1: 2)$ for $24 \mathrm{~h}$ at room temperature. Finally, the ICG@HSB-W5 thin film was obtained by soaking the HSB-W1 thin film into ICG aqueous solution for $24 \mathrm{~h}$ at room temperature. The membrane was washed several times with ultrapure water until no color was observed in the eluate and dried at room temperature.

\section{Characterization and instruments}

The morphologies of the thin films were characterized by scanning electron microscopy (SEM, SU-70/Hitachi S-4800) equipped with X-ray energy dispersive analysis. The X-ray diffraction (XRD) patterns of the thin films were characterized by X'Pert PRO (PANalytical, The Netherlands) instrument with $\mathrm{Cu}$ $\mathrm{Ka}$ radiation with $0.02^{\circ}$ step. The Fourier transform infrared (FTIR) spectra were performed on FTIR TENSOR 27 equipment by $\mathrm{KBr}$ pellets in the range of $400-4000 \mathrm{~cm}^{-1}$. The X-ray photoelectron spectroscopy (XPS, AXIS SUPRA, Krato) was used to identify the existence of ICG molecules. The $\mathrm{N}_{2}$ sorption isotherms were measured by Micromeritics instrument (3Flex) at $77 \mathrm{~K}$. The ultraviolet-visible (UV-vis) absorption spectra were recorded by a UV-vis spectrophotometer (Shimadzu UV-3600). The photoluminescence (PL) spectra and PL lifetime decay curves were recorded using a spectrophotometer (Edinburgh Instruments FLS920) with a 405-nm laser. The zeta potential was measured by Zetasizer Nano-ZS (Malvern). Infrared photos were taken by an infrared camera made by Foshan Teta Photographic Equipment Co. Ltd. at room temperature.

\section{Conductivity measurement}

Firstly, a pair of Ag electrodes were evaporated onto the surface of the thin film by the ZHD-300S film preparation system (BEIJING TECHNOL Co. Ltd.) with the current of $65 \mathrm{~A}$ for at least $30 \mathrm{~min}$. Then the alternating current (AC) impedance measurements were carried out by using a CHI 660D electrochemical workstation in the range from $1 \mathrm{MHz}$ to $100 \mathrm{~Hz}$ at the alternating current amplitude of $0.1 \mathrm{~V}$. Before the electrochemical measurement, the thin films were kept in a programmable temperature and humidity controller (BC1300, Shanghai YIHENG) for more than $2 \mathrm{~h}$ to stabilize. The infrared laser ( 808 $\mathrm{nm}-3 \mathrm{~W}-\mathrm{FC}$, Lasever Inc) and ultraviolet light sources (405 nm) were placed outside the controller to do the electrochemical impedance test under different lights. The optical density of the light at the samples' position was controlled at approximate $100 \mathrm{~mW} \mathrm{~cm}^{-2}$ and measured by PLS-MW2000 (PerfectLight).

The proton conductivity $(\sigma)$ is calculated based on the physical dimensions of the thin films according to the equation as follows:

$\sigma=\frac{L}{R S}$

where $L$ is the channel length $(300 \mu \mathrm{m}), R$ is the resistance value calculated from AC impedance spectra, and $S$ is the cross-section area of the flow transportation surface $(2 \mu \mathrm{m}$ (thickness) $\times 3 \mathrm{~mm}$ (width)) for the prepared thin films. The ON/OFF ratio of proton conductivity is defined as the average values of proton conductivity in dark to that under light and the proton con- 
ductivity values were measured after 5 min since the lights were turned on or off.

\section{RESULTS AND DISCUSSION}

\section{Characterization of the ICG@HSB-W5 thin films}

The HSB-W1 thin film was synthesized through a solid confinement conversion process according to the previous work [42]. The synthesis process of the ICG@HSB-W5 thin film is displayed in Scheme 1. HSB-W1 structure has three types of rhombic channels with diagonal distances of about $16.8 \times 12.5$, $15.5 \times 14.1$ and $14.3 \times 10.5 \AA^{2}$ to host guest ICG molecules (11.6 $\times 7.2 \times 20.7 \AA^{3}$ ) [29,37]. When dipped in green ICG solution, negatively charged ICG molecules [43] were attracted on the surface or into the pores of the positively charged HSB-W1 (zeta potential: $4.57 \mathrm{mV}$, Fig. S1a) through electrostatic interactions. The HSB-W5 structure with smaller channel was formed gradually meantime and ICG molecules were encapsulated in the pores tightly. After loading of negatively charged ICG, the zeta potential of ICG@HSB-W5 became negative (zeta potential: $-16.5 \mathrm{mV}$, Fig. S1b).

By varying the concentration of the ICG in aqueous solution, the content of ICG encapsulated in the HSB-W5 thin film can be adjusted accordingly. The concentrations of ICG aqueous solution used to decorate the HSB-W5 thin film are $0.005,0.01,0.05$, $0.1,0.2$ and $0.5 \mathrm{mg} \mathrm{mL}^{-1}$, respectively. In addition, according to the change of ICG concentration before and after being soaked with HSB-W 1 thin films, the ICG content of each HSB-W5 thin film and the wight ratios of ICG to HSB-W5 of the thin films can be calculated through the UV-vis absorption standard curve method (Fig. S2). The obtained pure HSB-W5 thin film and a series of ICG@HSB-W5 thin films are denoted as S-0 and S-1, S2, S-3, S-4, S-5, S-6, respectively (Table S1).

The surface and cross-sectional SEM images of the assynthesized thin films are shown in Fig. 1a-e and Fig. S3. It is clear that all the thin films have similar surface morphologies

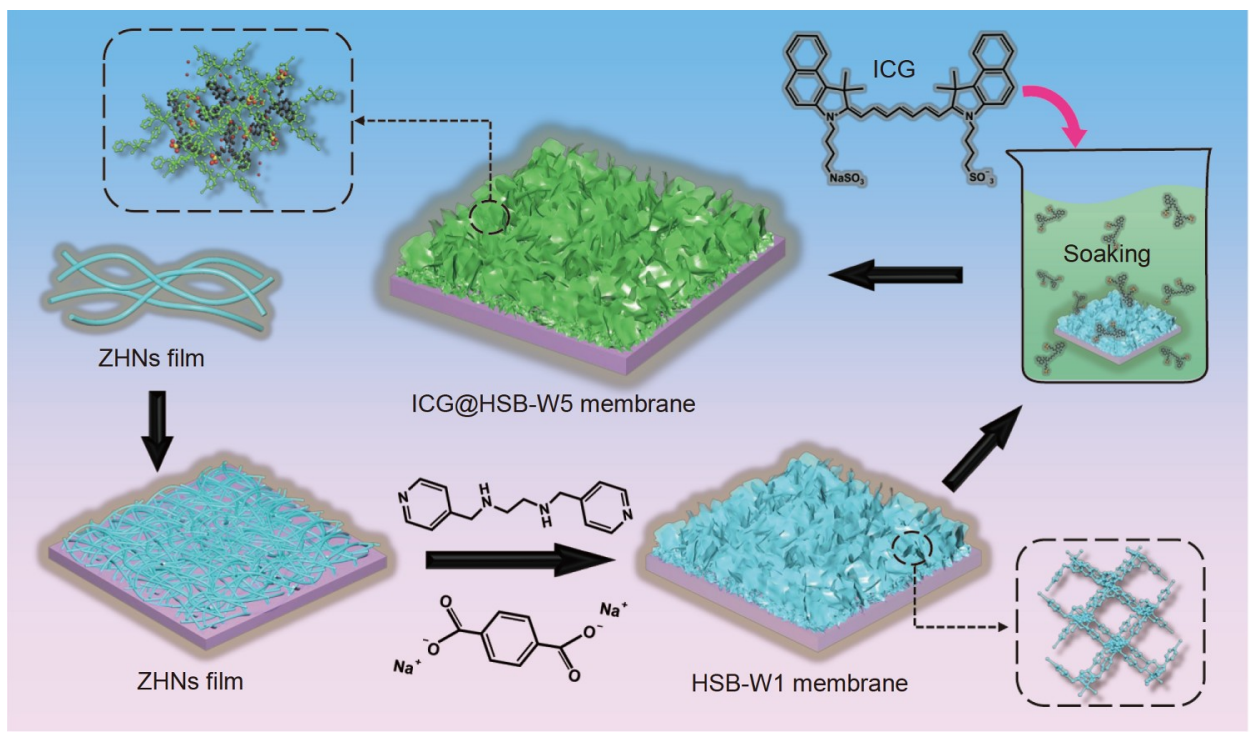

Scheme 1 Illustration of the synthesis process of the ICG@HSB-W5 thin film.
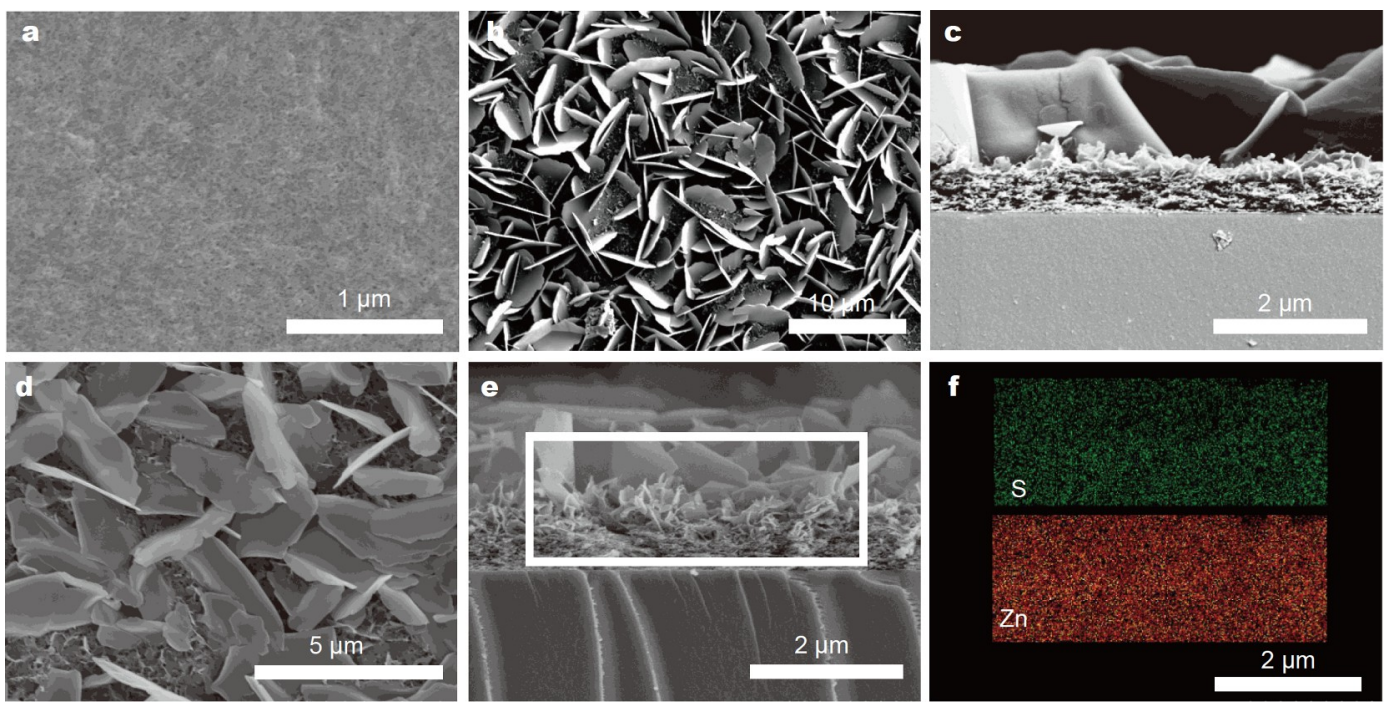

Figure 1 (a) SEM image of the ZHNs. Surface and cross-sectional SEM images: (b, c) S-0; (d, e) S-4. (f) S (green) and Zn (red) EDS mapping images from the zone marked in (e). 
with different sizes of leaflike nanosheets randomly stacking. The water-triggered structural conversion from HSB-W1 to HSB-W5 and the encapsulation of ICG in the HSB-W5 framework do not influence the leaflike morphologies. From the crosssectional images (Fig. 1c, e), the thicknesses of S-0 and S-4 are both approximately $2 \mu \mathrm{m}$, which is used to calculate the proton conductivity. The cross-sectional energy dispersive spectroscopy (EDS) mapping images (Fig. 1f) show a uniform distribution of $\mathrm{S}$ and $\mathrm{Zn}$, indicating that ICG is uniformly distributed in the thin film and evenly encapsulated into the crystal of HSB-W5. The XRD patterns (Fig. 2a and Fig. S4a) show that the HSB-W1 phase successfully forms and almost all of the HSB-W1 is transformed into HSB-W5 with sharp diffraction peaks at $6.6^{\circ}$ and $9.6^{\circ}[29,31]$. Besides, the ICG powder has no distinct peak and does not affect the formation of HSB-W5 crystals.

The ICG@HSB-W5 thin films were also characterized by FTIR spectroscopy and compared with the pristine HSB-W5 and ICG, respectively. As shown in Fig. $2 \mathrm{~b}$ and Fig. $\mathrm{S} 4 \mathrm{~b}$, the characteristic peak of $\mathrm{SO}_{3}^{-}$stretching at $1049 \mathrm{~cm}^{-1}$ indicates the presence of ICG inside the ICG@HSB-W5 thin films [28]. HSB-W5 and HSB-W1 have the same FTIR spectrum because of their common functional groups. As the ICG content in the thin film increases, the intensity of peak at $1424 \mathrm{~cm}^{-1}$ becomes stronger. Besides, the peak at $1010 \mathrm{~cm}^{-1}$ existing in ICG@HSB-W5 thin films is different from the peak at $1018 \mathrm{~cm}^{-1}$ of HSB-W5 and the peak at $1005 \mathrm{~cm}^{-1}$ of ICG. This shift can be ascribed to the interaction of $\mathrm{Zn}^{2+}$ with part of the sulfonate groups of ICG molecules [44]. Furthermore, XPS results (Fig. 2c, Fig. S5a, b) show that $\mathrm{S}$ exists in $\mathrm{S}-4$ but does not appear in S-0, confirming the adsorption of ICG in the HSB-W5 thin film. The $\mathrm{N}_{2}$ adsorption-desorption isotherms and pore structures (Fig. 2d, Fig. S5c) of the S-0 and S- 4 indicate that the Brunauer-EmmettTeller (BET) surface area $\left(22.709 \mathrm{~m}^{2} \mathrm{~g}^{-1}\right)$ and pore volume $\left(0.2398 \mathrm{~cm}^{3} \mathrm{~g}^{-1}\right)$ of $\mathrm{S}-4$ are both smaller than those of S-0 $\left(25.030 \mathrm{~m}^{2} \mathrm{~g}^{-1}\right.$ and $\left.0.2590 \mathrm{~cm}^{3} \mathrm{~g}^{-1}\right)$. This further confirms the incorporation of ICG molecules into the pores of HSB-W5. From the above results, it is proved that ICG molecules have been successfully introduced into the HSB-W5 thin film.

The UV-vis absorption spectra were carried out to explore the optical property of the thin films. According to Fig. 2e and Fig. S5d, S-4 has a similar behavior to the ICG aqueous solution between $600-850 \mathrm{~nm}$ with a wider absorption range. The strong light absorption in this range provides a promising possibility to convert the near-infrared (NIR) light into heat. Meanwhile, the main absorption peaks of ICG have shifted from 715 and $776 \mathrm{~nm}$ to 737 and $812 \mathrm{~nm}$ in S-4, which might be related to the weak interaction between $\mathrm{Zn}^{2+}$ and ICG molecules [45]. In contrast, S0 has little absorption of NIR light compared with S-4 and other ICG-containing thin films. At the wavelength of approximately $405 \mathrm{~nm}, \mathrm{~S}-4$ also has an obvious absorption due to the host HSBW5, which contributes to the photothermal conversion under 405-nm light. The PL spectra of the thin films (Fig. 2f, Fig. S6a) show that the largest peak of S-0 is clearly observed at $473 \mathrm{~nm}$ when excited at $405 \mathrm{~nm}$, which is derived from the hydrogenated Schiff base ligand [29,30]. After loading ICG, the fluorescence intensity of the S- 4 becomes much lower than that of S-0 (almost one-tenth of S-0), and no obvious PL peak (at approximately $820 \mathrm{~nm}$ ) of ICG (Fig. S6b, c) is observed. The blue fluorescence of HSB-W5 is quenched after the incorporation of ICG due to the possible energy transfer between ICG and HSB-W5. The dramatic decrease of PL intensity demonstrates that most of the absorption optical energy may be converted into heat energy (see detail in next section). Furthermore, it is reasonable to speculate that the phenomenon of "aggregation-caused quenching (ACQ)" occurs when large numbers of ICG molecules are embedded in the HSB-W5 thin film and the ACQ phenomenon contributes to heat generation as well [45]. These are another two factors to reversibly control the proton conductivity by light in the thin films.

To examine the photothermal conversion ability of the thin films, S-0 and S-4 were continuously irradiated by $808-\mathrm{nm}$ $\left(100 \mathrm{~mW} \mathrm{~cm}^{-2}\right)$ laser and $405-\mathrm{nm}\left(100 \mathrm{~mW} \mathrm{~cm}^{-2}\right)$ laser, respectively, and the corresponding temperatures were recorded with an infrared thermal camera. As shown in Fig. S7a, the average temperatures within the dotted line circles increase at first and reach to plateau at about the $5^{\text {th }}$ minute after exposure to the lasers. The temperature elevations of S-0 under 808 and $405 \mathrm{~nm}$ are not obvious until the $10^{\text {th }}$ minute (Fig. S7b, d). But the temperature of S-4 improves from room temperature to $44.3^{\circ} \mathrm{C}$ under $808 \mathrm{~nm}$ and $41.4^{\circ} \mathrm{C}$ under $405 \mathrm{~nm}$ (Fig. S7c, e), much higher than those of S-0. Furthermore, a continuous illumination test was performed in the sequence of dark, $808 \mathrm{~nm}, 808 \mathrm{~nm}$ plus $405 \mathrm{~nm}, 808 \mathrm{~nm}$, and dark again to investigate the temperature variation at the working state (Fig. 3a, Fig. S7f-j). The temperatures under $808 \mathrm{~nm}$ simply at the $9^{\text {th }}$ minute $\left(41.5^{\circ} \mathrm{C}\right)$ and $30^{\text {th }}$ minute $\left(43.4^{\circ} \mathrm{C}\right)$ are close. After irradiated by $808 \mathrm{~nm}$ plus $405 \mathrm{~nm}$, the temperature of S- 4 increases to $67.3^{\circ} \mathrm{C}$ efficiently with a much brighter surface color. These stair-stepping plots confirm that the optical energy of the lasers is converted into thermal energy successfully, causing obvious temperature elevation in S-4.

\section{Proton conductivity and photoswitching performance}

To evaluate the proton transporting performance in the ICG@HSB-W5 thin films, the proton conductivities of the thin films were investigated by electrochemical impedance (EIS). The samples were measured at a constant temperature and $\mathrm{RH}$. If not specifically mentioned, the proton conductivity was tested in the dark condition. In Fig. S8a, b, the proton conductivity increases from S- 0 to S-4 and reaches to relatively stable values of S-5 and S-6, which relates to their ICG content variation. It can be found that the proton conductivity $\left(1.91 \times 10^{-4} \mathrm{~S} \mathrm{~cm}^{-1}\right)$ of S-4 is 25 times higher than that $\left(7.54 \times 10^{-6} \mathrm{~S} \mathrm{~cm}^{-1}\right)$ of $\mathrm{S}-0$ at $55^{\circ} \mathrm{C}$ and $95 \% \mathrm{RH}$ due to the introduction of ICG molecules with numerous sulfonate groups. The influence of temperature and $\mathrm{RH}$ on the proton conductivities of the resulting thin films are shown in Fig. S8c-f. The proton conductivity of S-4 increases from $1.19 \times 10^{-4}$ to $6.85 \times 10^{-4} \mathrm{~S} \mathrm{~cm}^{-1}$ with the temperature increasing from 45 to $85^{\circ} \mathrm{C}$ at $95 \% \mathrm{RH}$. Moreover, the proton conductivity of S-4 heavily depends on ambient humidity, increasing from $1.17 \times 10^{-7}$ to $1.91 \times 10^{-4} \mathrm{~S} \mathrm{~cm}^{-1}$ when the humidity increases from $55 \%$ to $95 \% \mathrm{RH}$ at $55^{\circ} \mathrm{C}$, which improves by more than 1000 times. The increasing water molecules inside the channels promote the formation of the hydrogen-bond network and introduce more proton carriers for proton transportation $[28,46]$.

Furthermore, the proton conducting mechanism was explored by measuring the variation of proton conductivities under different temperatures. As shown in Fig. S9, the activation energies have a decreasing tendency with the increase of ICG content in the thin films. The proton conductions in the samples follow an Arrhenius-like behavior with activation energies ranging from 

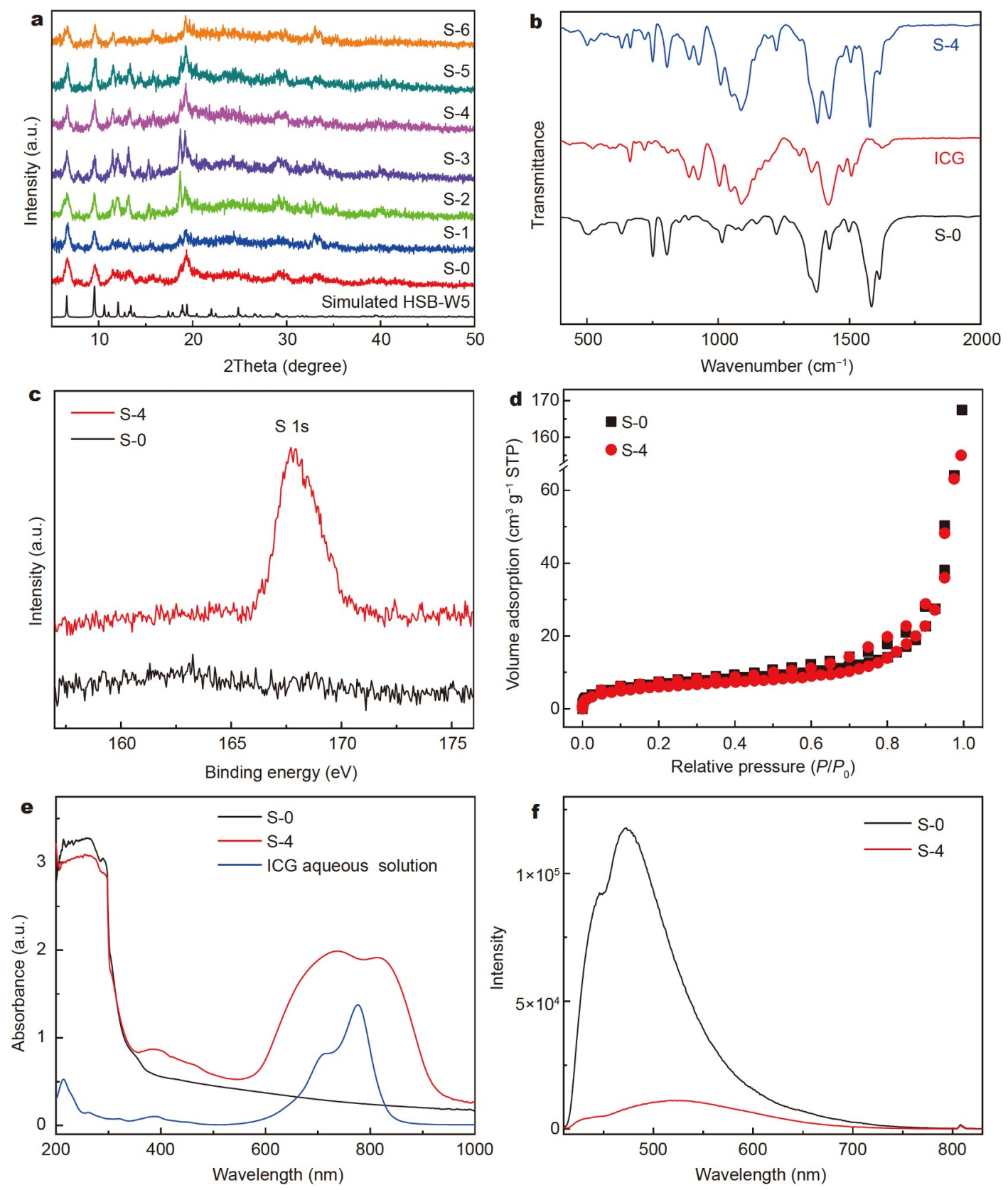

Figure 2 (a) XRD patterns of the samples. (b) FTIR spectra of S-0, S-4 and ICG. (c) S 1 s XPS of the S- 0 and S-4. (d) N $\mathrm{N}_{2}$ sorption isotherms at 77 K of S-0 and S-4. (e) UV-vis absorption spectra of S-0, S-4, and ICG aqueous solution. (f) PL spectra of the S-0 and S-4 excited at $405 \mathrm{~nm}$.

0.46 to $0.75 \mathrm{eV}$ at $95 \% \mathrm{RH}$. The activation energy of the pure HSB-W5 thin film S-0 $(0.75 \mathrm{eV})$ is much higher than $0.40 \mathrm{eV}$, indicating that proton conduction in the S-0 obeys the vehicle mechanism [47]. This result suggests that some of the water molecules in the S-0 have the possibility to accept protons and transport protons through direct self-diffusion in the thin film. After ICG molecules are incorporated into the thin film, the activation energy of S- 4 decreases to $0.48 \mathrm{eV}$, which is very close to the range for the Grotthuss mechanism $(\leq 0.4 \mathrm{eV})$ [48]. It indicates that proton conduction in the S-4 is easier than that in S-0. Given that hydrogen-bond cleavage needs a low energy $(\sim 0.11 \mathrm{eV})$ and lots of sulfonate groups and water molecules exist in S-4, sufficient hydrogen-bond network formed by ICG and water molecules in the MOF channels makes protons hop along the network through protonation and deprotonation of water molecules, providing fast and efficient proton conductive pathways. This means Grotthuss is the major proton transport mechanism in S-4 while partial vehicle mechanism may also occur.

The photoswitching performance of the thin films is a critical index for logic gate construction. According to our previous work on photoswitching proton conduction in MOF thin films, a relatively low temperature is more suitable for photoswitching performance and highlights the photothermal effect in a humid condition $[27,28]$. As mentioned above, the highest proton conductivity was achieved at $95 \% \mathrm{RH}, 22$ times higher than that under $85 \% \mathrm{RH}$. Therefore, the temperature of $55^{\circ} \mathrm{C}$ and the humidity of $95 \% \mathrm{RH}$ were applied in this study. The behaviors of 

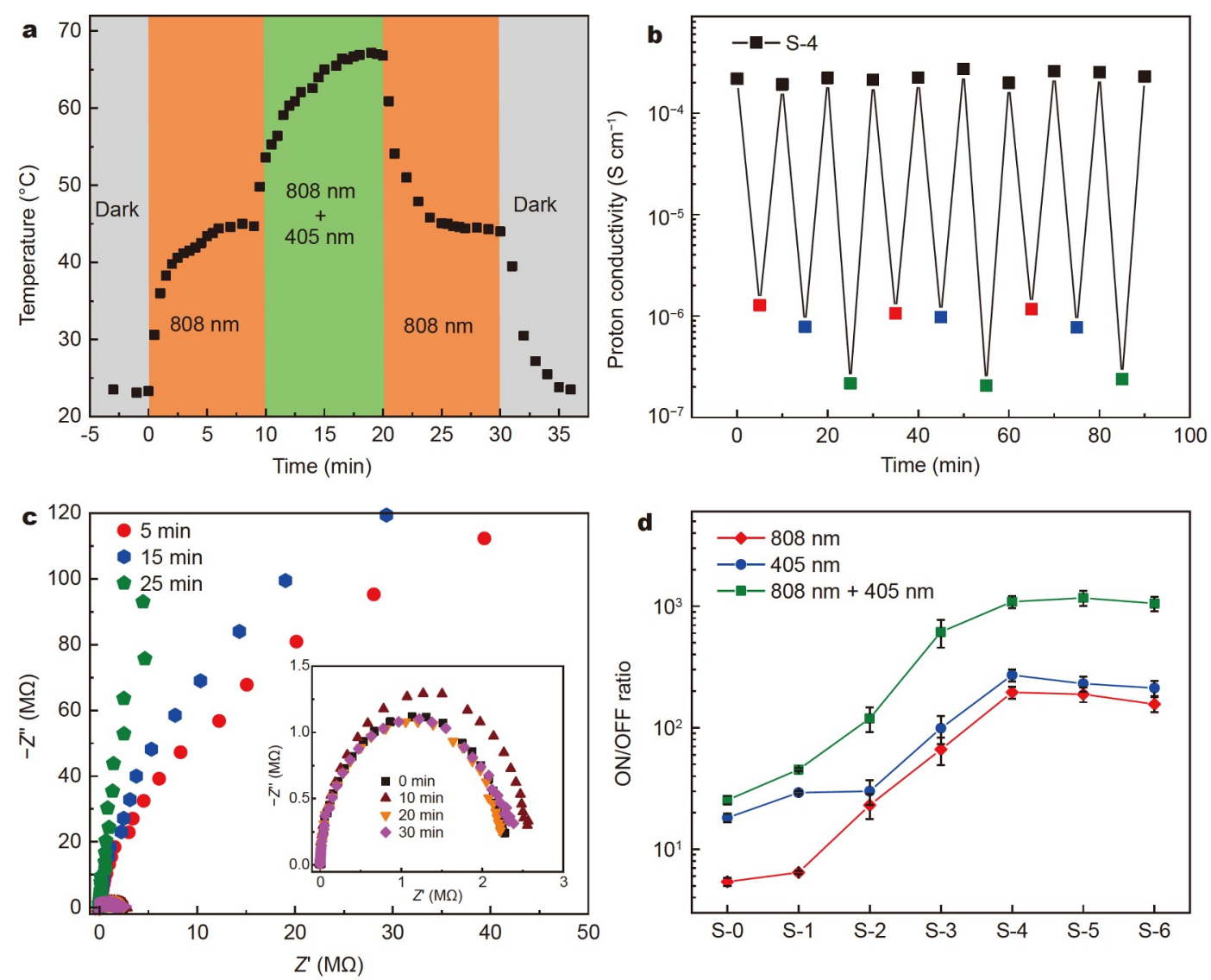

Figure 3 (a) Temperature elevation curve of S-4 under 808 - and 405 -nm lights. The room temperature is $22.3-24.5^{\circ} \mathrm{C}$ when the measurements were carried out. (b) Proton conductivity of S- 4 with light off (black point) and on (red point: $808 \mathrm{~nm}$; blue point: $405 \mathrm{~nm}$; green point: 808 and $405 \mathrm{~nm}$ simultaneously) at $55^{\circ} \mathrm{C}$ and $95 \% \mathrm{RH}$. (c) Corresponding Nyquist plots of (b). (d) ON/OFF ratios of the samples with different kinds of light at $55^{\circ} \mathrm{C}$ and $95 \%$ RH.

the thin films under light and dark in a consecutive process are displayed in Fig. 3b, c and Fig. S10. In the cycled measurement graphs, four different color points (black, red, blue and green) mean that they were measured under dark, 808, $405 \mathrm{~nm}$ and both of them at the same time, respectively. The ON/OFF ratio is used to evaluate the photoswitching performance and the $\mathrm{ON} /$ OFF ratio variations are shown in Fig. $3 \mathrm{~d}$. In the dark, the proton conductivity of S-4 is $2.18 \times 10^{-4} \mathrm{~S} \mathrm{~cm}^{-1}$ but it decreases to $1.28 \times 10^{-6} \mathrm{~S} \mathrm{~cm}^{-1}$ greatly once exposed to $808-\mathrm{nm}$ light, resulting in an ON/OFF ratio of 195.4. Turning off 808-nm light, the proton conductivity almost restores to the initial value within $5 \mathrm{~min}$. Then upon switching to $405-\mathrm{nm}$ light immediately, the proton conductivity falls sharply to $7.81 \times 10^{-7} \mathrm{~S} \mathrm{~cm}^{-1}$ with an ON/OFF ratio of 271.0, larger than that under $808-\mathrm{nm}$ light. When 808- and 405-nm lights are shone on S-4 together, the ON/OFF ratio soars to 1036.3 and the proton conductivity $\left(2.16 \times 10^{-7} \mathrm{~S} \mathrm{~cm}^{-1}\right)$ gets much lower. The dramatic changes can be seen visually in the corresponding Nyquist plots of S-4 (Fig. 3c). The speed of the optical-controlled switch is also important. However, limited by the test time of EIS measurement, it is hard to calculate the accurate switch speed value. Actually, the response time of the proton conductivity is much less than $5 \mathrm{~min}$. In addition, the decrease and recovery of the proton conductivity can be realized quickly and maintain the behaviors throughout the whole testing process for $90 \mathrm{~min}$. Hence, the thin film shows good cycling and recovery performances. Without ICG molecules, S-0 exhibits a smaller change under alternating lasers and its ON/OFF ratios in different situations (5.4, 18.2 and 25.4, under $808,405 \mathrm{~nm}$ and both of them respectively) are far less than those of S-4. As ICG content increases, ON/OFF ratios of the three illuminating states gradually increase from S-1 to S-4 and step on a plateau in S-5 and S-6. Eventually, there are four stable distinguished states of the proton conductivity of S- 4 that can be reversibly optical-controlled by external lasers. For use as logic gates, a proton conduction system should have at least two stable states, which can be reversibly converted by external stimuli [49]. Therefore, it is possible for constructing proton logic gates with laser as the input and proton conductivity as the output.

The proton conducting mechanism in response to the two lasers and the corresponding proton logic gates are shown in Scheme 2. The sulfonate groups and water molecules form the hydrogen-bond network together and offer the proton transport highways in ICG@HSB-W5 thin films in the dark condition. Meanwhile, some proton carriers like $\mathrm{H}_{3} \mathrm{O}^{+}$diffuse directly in the thin film. When exposed to $808 \mathrm{~nm}$, ICG absorbs the NIR light strongly and converts optical energy into heat immediately through the photothermal conversion effect. In the proton conductive channels, local heating causes some high-temperature zones where the temperature is higher than the ambient temperature and some water molecules are forced to leave from the channels. Accordingly, the hydrogen-bond network is destroyed and proton carriers decrease. Thus, the proton transport paths are limited, narrowed or even cut off at the high- 


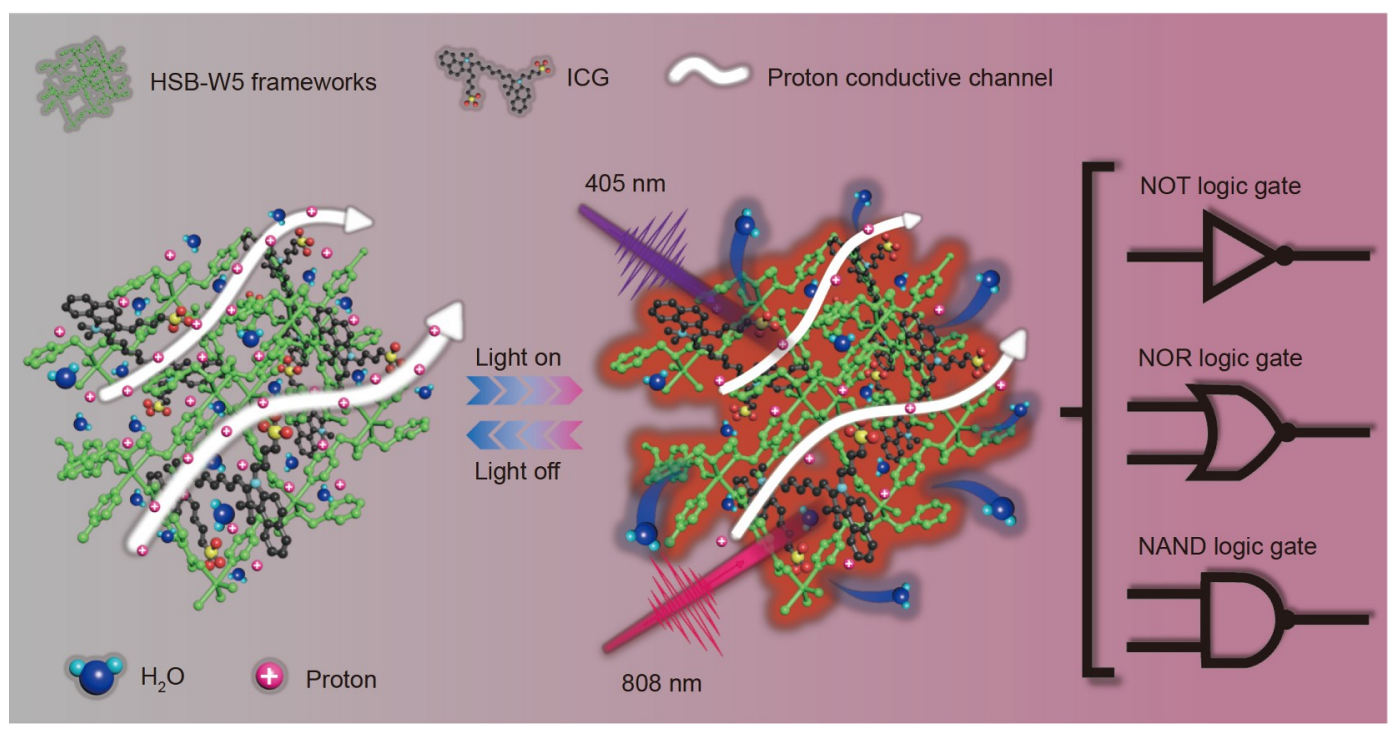

Scheme 2 Illustration of the proton transport mechanism under 808- and 405-nm lasers through the photothermal effect in the ICG@HSB-W5 thin films and the corresponding logic gates.

temperature zones, leading to a low proton conductivity. The proton conductivity is restored later when the light is switched off owing to regaining water molecules from other regions of the thin film and high humidity surroundings to rebuild the hydrogen-bond network. The situation is similar when we switch from 808 to $405 \mathrm{~nm}$ but the thermal energy in this case mainly comes from the host MOF HSB-W5 through fluorescence quenching rather than ICG. When 808- and 405-nm lights are both turned on, the photothermal conversion from ICG and fluorescence quenching from HSB-W5 occur at the same time and generate more powerful heat in a short time to heat the water molecules, resulting in a much lower proton conductivity than the single ones. In summary, these endow the S-4 with an excellent photothermal conversion effect to trigger the protonbased switch.

Notably, how does the thermal energy generate to switch the proton conductivity deserves further study. ICG molecule has a $\pi$-conjugated structure, making it have strong absorption for NIR light in the wavelength range of $600-850 \mathrm{~nm}$. After being irradiated by NIR light, the electrons in the dye molecule are excited to transform from the ground state to the excited state. When the transitioning electrons return to the ground state, their energy will be released in the form of heat and fluorescence [50-52]. First of all, the photothermal effect of ICG is a simple and direct way to generate heat under $808-\mathrm{nm}$ light. Part of the absorbed NIR light energy is converted into heat energy directly. In the meantime, some absorbed energy will be released in the form of fluorescence but the PL peak at $820 \mathrm{~nm}$ of the ICG is not observed in the ICG@HSB-W5 thin films. Considering that the concentration of ICG in the thin film is much larger than that in aqueous solution, the phenomenon of ACQ tends to happen and provides a nonradiative relaxation process. For infrared fluorescence imaging, ACQ is a negative factor [53,54]. But for photothermal conversion, ACQ is beneficial to heat generation and offers a new way to realize light-to-heat conversion. In addition, the fluorescence of HSB-W5 exited at $405 \mathrm{~nm}$ is quenched by ICG molecules in the ICG@HSB-W5 thin films, causing another photothermal conversion approach. Compared with ICG@HSB-W5 thin films, the fluorescence of S-0 is very strong due to the organic linkers. Every $\mathrm{Zn}$ atom is five-coordinated in HSB-W5 with a distorted trigonal bipyramidal $\left[\mathrm{ZnN}_{3} \mathrm{O}_{2}\right]$ geometry, which comes from HSB-W1 with a sixcoordinated slightly distorted octahedral coordination configuration $\left[\mathrm{ZnN}_{4} \mathrm{O}_{2}\right][29,31]$. The special structure leaves an opportunity to form the bond between the sulfonate groups of ICG and $\mathrm{Zn}^{2+}$ centered atom. The interactions between $\mathrm{Zn}^{2+}$ and the embedded ICG creates an extra electron transition pathway, possibly leading to the lower fluorescence intensity of ICG@HSB-W5 thin films. To further confirm the fluorescence quenching mechanism discussed above, PL lifetime curves were measured (Fig. S11) and the fitting parameters of the PL lifetime decay are provided in Table S2. The average PL lifetime (3.63 ns) of S-4 is shorter than that of S-0 (3.90 ns).

In order to investigate the sequence of irradiation on the cycled and recovery property of proton conductivity, a consecutive cycled measurement (Fig. S12a) was carried out with different sequences of the 808- and 405-nm lasers. The proton conductivities of S- 4 under dark, $808,405 \mathrm{~nm}$ and two lasers simultaneously have similar behaviors with Fig. 3 b. This result demonstrates that random combinations of the lasers do not affect the sample's response to lights. Besides, the long-term durability of the thin film is also investigated. After being kept at room temperature for three months, the proton conductivity $\left(1.64 \times 10^{-4} \mathrm{~S} \mathrm{~cm}^{-1}\right)$ of S-4 decreases slightly (Fig. S12b) compared with the initial state but the four states are still recognized easily, indicating a good durability of the thin film.

All of the advantages, including the simple infiltration synthesis process, excellent photothermal conversion ability, rapid response to light, good recovery stability, and nice durability, make the ICG@HSB-W5 thin film a great signal switch candidate for constructing logic gates.

\section{Construction of logic gates}

In terms of the above analysis, some basic logic gates are designed according to Boolean logical library. In this work, the electrical signal, proton conductivity of S- 4 at $55^{\circ} \mathrm{C}$ and $95 \% \mathrm{RH}$, is used directly as the output with different thresholds. Considering that the proton conductivity of S- 4 can be altered by 
808- and 405-nm lasers, the lasers are used as two inputs $\left(\mathrm{I}_{1}=\right.$ $808 \mathrm{~nm}, \mathrm{I}_{2}=405 \mathrm{~nm}$ ) to construct the logic gates. The presence and absence of the two inputs $\mathrm{I}_{1}$ and $\mathrm{I}_{2}$ are defined as " 1 " and "0", respectively. Two thresholds of proton conductivity $(\sigma)$ are applied in different logic gates flexibly according to the actual demand. The turn-on state is defined as output " 1 " when it exceeds the threshold limit of proton conductivity and the turnoff state is defined as output " 0 " when it fails to exceed the threshold limit.

In NOT logic, the output is the opposite of the input. NOT logic gate is the simplest gate to design using only one input and getting one output as well. But it is an important member of the basic logic gates and many complex logic calculations must rely on it [23]. Because of the high ON/OFF ratio of S-4 under 808or 405-nm light, a NOT logic gate is realized based on S-4 (Fig. 4a). In this case, the proton conductivity above or below Threshold-1 $\left(\sigma=10^{-5} \mathrm{Scm}^{-1}\right)$ is defined as "1" or " 0 ", respec-

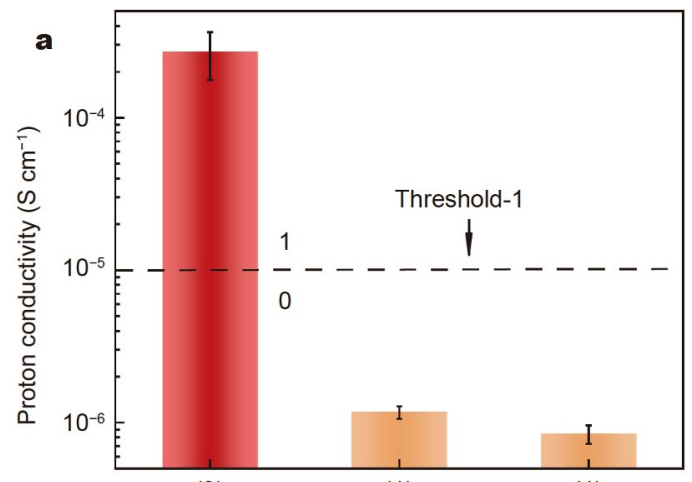

(0)

(1)
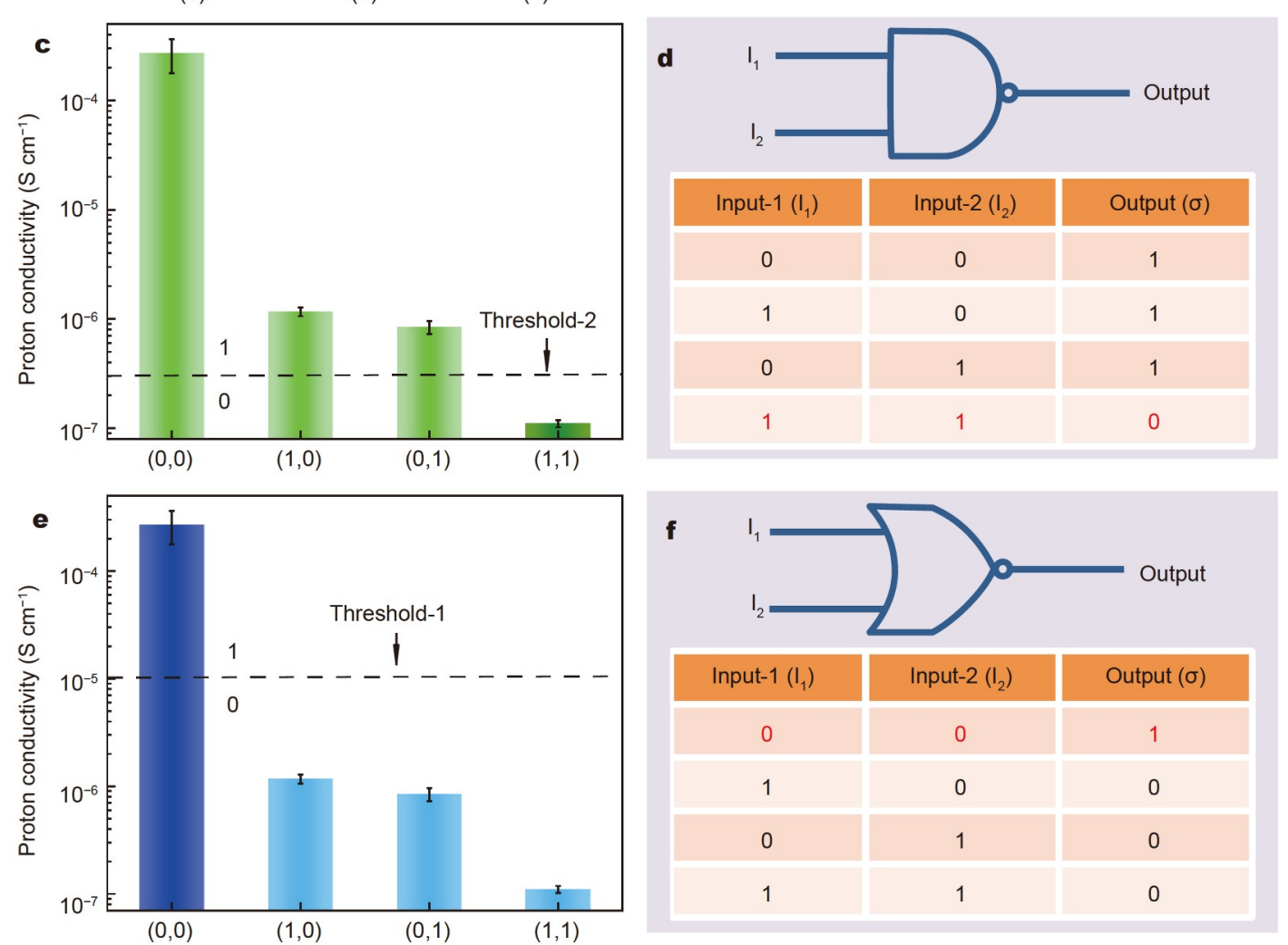

tively. The input is recorded as " 1 " when $I_{1}$ is switched on and the proton conductivity decreases to a low value under Threshold-1, resulting in the output of " 0 ". It is the same result from either photothermal conversion effect of ICG or fluorescence quenching of the MOF hurdles the proton transportation remarkably. Evidently, the switch effect of laser on the proton conductivity is in line with the principle of the NOT gate. The electronic equivalent circuit and the truth table of NOT gate are also shown in Fig. 4 b. proton conductivity is distinct enough from the aforesaid three of proton conductivity suit for construction of NAND logic gate (Fig. 4c) with the Threshold-2 $\left(\sigma=2 \times 10^{-7} \mathrm{~S} \mathrm{~cm}^{-1}\right)$. The input of S-4 displays four possible combinations of $(0,0),(1,0),(0,1)$ and

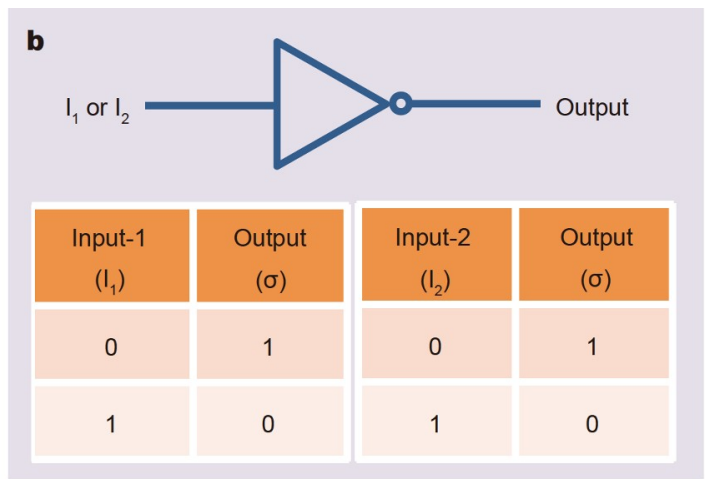
when $I_{2}$ is used as the input separately. The thermal generation

Exposed to the two lasers simultaneously, the decrease of the states, which is the foundation of the other two logic gates. When $I_{1}$ and $I_{2}$ are used as inputs at the same time, the changes

Figure 4 (a) Proton conductivity of S-4 with $\mathrm{I}_{1}$ or $\mathrm{I}_{2}$ as input with Threshold-1. (b) NOT logic gate circuit and the truth table. (c) Proton conductivity of S-4 with $\mathrm{I}_{1}$ and $\mathrm{I}_{2}$ as inputs with Threshold-2. (d) NAND logic gate and the truth table. (e) Proton conductivity of S-4 with $\mathrm{I}_{1}$ and $\mathrm{I}_{2}$ as inputs with Threshold-1. (f) NOR logic gate and the truth table. They were all measured at $55^{\circ} \mathrm{C}$ and $95 \% \mathrm{RH}$. 
$(1,1)$. Only in the presence of $I_{1}$ and $I_{2}$ (input $=(1,1)$ ), the proton conductivity dives dramatically to $1 \times 10^{-7} \mathrm{~S} \mathrm{~cm}^{-1}$ level, yielding an output of " 0 ". The absence of any input or both of them leads to an output of " 1 ". The logic gate circuit and the truth table of NAND gate are also depicted in Fig. 4 d.

The NOR logic gate is the inverse of the OR gate and output " 1 " is observed only in the absence of any input. Guided by this principle, a NOR logic gate is constructed based on S-4 with the Threshold-1. What it can be drawn from Fig. 4e is that only when $I_{1}$ and $I_{2}$ are both absent (input $=(0,0)$ ), does the proton conductivity maintain its initial value, thus resulting in an output of " 1 ". When both inputs are present (input $=(1,1)$ ) or either input is present (input $=(1,0)$ or $(0,1))$, the proton conductivity is no more than Threshold-1 (output $=0$ ). Hence, the effect of the lasers on the proton conductivity is consistent with the design principle of NOR logic gate. The logic gate circuit and the truth table are shown in Fig. 4f.

From the discussion above, it is evident that the proton conductivity of S- 4 can be tuned by using different lasers and different logic operations can be implemented based on the different combinations of inputs. To accomplish a sophisticated molecular device, a multi-input logic operation was constructed by using basic logic gates and universal logic gates. As shown in Fig. S13, a multi-input logic operation is formed by using NOT and NAND gates as input combination for a next basic AND gate. $I_{1}$ and $I_{2}$ are still used as logic inputs. The absence of any input does not alter the proton conductivity of S-4 (output $=1$ ). The presence of $I_{1}$ or $I_{2}$ in the NAND gates with no input of NOT gate can lead to a high level of proton conductivity (output $=1$ ). The output is " 0 " in other combinations of three inputs. The truth table is shown in Fig. S13 as well.

Moreover, we tried to apply the S-4 thin film to control a red light-emitting diode (LED) lamp with the help of an amplification circuit under $6 \mathrm{~V}$ direct voltages (Fig. S14a), which was constructed by a bipolar junction transistor and can amplify the current by 100 times. The thin film was placed in the temperature and humidity controller at $55^{\circ} \mathrm{C}$ and $95 \% \mathrm{RH}$ and connected with the amplification circuit outside by wires. The LED lamp was turned on (output $=1$ ) when the S-4 thin film was stored in the dark condition, whereas the lamp was turned off (output $=0$ ) after irradiated by 808, 405, 808 and $405 \mathrm{~nm}$, respectively (Fig. S14b-e). It fits with the principle of NOT and NOR logic gates with Threshold-1 in this circumstance. The large difference in the proton conductive resistance before and after illumination (Fig. 3c) is vital for this process. The current through the LED lamp can reach $41.2 \mu \mathrm{A}$ in the dark with the aid of the amplification circuit, which is large enough to light up the lamp. After illumination, the current through the LED lamp is too small to light up the lamp even after the amplification (Table S3). Similarly, the NAND logic gate can be realized as well when we change to Threshold-2. Therefore, it is possible to combine this thin film with existent electronic devices on account of the electrical output.

\section{CONCLUSIONS}

In conclusion, we developed photo-controlled proton logic gates by introducing ICG molecules into HSB-W5 thin film simply. The sulfonate groups of ICG significantly improve the proton conductivity of ICG@HSB-W5 thin film in the dark by generating a hydrogen-bond network and introducing proton carriers. Upon 808- and 405-nm lasers, the photothermal conversion effect of ICG and the fluorescence quenching of HSB-W5 endow the thin film with outstanding switching property. The S-4 possesses not only clearly distinguished ON/ OFF ratios, but also fast response upon the two lasers at $55^{\circ} \mathrm{C}$ and $95 \%$ RH. Typically, three basic logic gates (NOT, NAND and NOR) were successfully constructed with flexible thresholds based on the thin film. More importantly, the output proton conductivity can be detected easily by other traditional logic gates or electronic devices without further signal transformation. This is a new attempt on design of molecular logic gate based on MOF and it provides a possibility for proton-conducting fieldeffect transistors, demonstrating a great potential for the molecular computing.

Received 16 August 2021; accepted 18 September 2021; published online 17 November 2021

1 Escorihuela J, Narducci R, Compañ V, et al. Proton conductivity of composite polyelectrolyte membranes with metal-organic frameworks for fuel cell applications. Adv Mater Interfaces, 2018, 1801146

2 Meng X, Wang HN, Song SY, et al. Proton-conducting crystalline porous materials. Chem Soc Rev, 2017, 46: 464-480

3 Kanj AB, Chandresh A, Gerwien A, et al. Proton-conduction photomodulation in spiropyran-functionalized MOFs with large on-off ratio. Chem Sci, 2020, 11: 1404-1410

4 Karim MR, Hatakeyama K, Koinuma M, et al. Proton conductors produced by chemical modifications of carbon allotropes, perovskites and metal organic frameworks. J Mater Chem A, 2017, 5: 7243-7256

5 Amdursky N, Wang X, Meredith P, et al. Long-range proton conduction across free-standing serum albumin mats. Adv Mater, 2016, 28: 2692-2698

6 Guo Y, Peng X. Mass transport through metal organic framework membranes. Sci China Mater, 2018, 62: 25-42

7 Lim DW, Kitagawa H. Proton transport in metal-organic frameworks. Chem Rev, 2020, 120: 8416-8467

8 He S, Wang $\mathrm{H}$, Zhang $\mathrm{C}$, et al. A generalizable method for the construction of MOF@polymer functional composites through surfaceinitiated atom transfer radical polymerization. Chem Sci, 2019, 10: $1816-1822$

9 Zhou HC, Long JR, Yaghi OM. Introduction to metal-organic frameworks. Chem Rev, 2012, 112: 673-674

10 Li PZ, Wang XJ, Liu J, et al. A triazole-containing metal-organic framework as a highly effective and substrate size-dependent catalyst for $\mathrm{CO}_{2}$ conversion. J Am Chem Soc, 2016, 138: 2142-2145

11 Khezri B, Pumera M. Metal-organic frameworks based nano/micro/ millimeter-sized self-propelled autonomous machines. Adv Mater, 2019, 31: 1806530

$12 \mathrm{Gu} \mathrm{J}$, Fan H, Li C, et al. Robust superhydrophobic/superoleophilic wrinkled microspherical MOF@rGO composites for efficient oil-water separation. Angew Chem Int Ed, 2019, 58: 5297-5301

13 Kanda S, Yamashita K, Ohkawa K. A proton conductive coordination polymer. I. [N,N'-bis(2-hydroxyethyl)dithiooxamido]copper(II). Bull Chem Soc Jpn, 1979, 52: 3296-3301

14 Horike S, Umeyama D, Kitagawa S. Ion conductivity and transport by porous coordination polymers and metal-organic frameworks. Acc Chem Res, 2013, 46: 2376-2384

15 Ramaswamy P, Wong NE, Shimizu GKH. MOFs as proton conductors —challenges and opportunities. Chem Soc Rev, 2014, 43: 5913-5932

16 Pili S, Argent SP, Morris CG, et al. Proton conduction in a phosphonate-based metal-organic framework mediated by intrinsic "free diffusion inside a sphere". J Am Chem Soc, 2016, 138: 6352-6355

17 Müller K, Helfferich J, Zhao F, et al. Switching the proton conduction in nanoporous, crystalline materials by light. Adv Mater, 2018, 30: 1706551

18 Heinke L, Wöll C. Surface-mounted metal-organic frameworks: Crystalline and porous molecular assemblies for fundamental insights and advanced applications. Adv Mater, 2019, 31: 1806324 
19 Nagarkar SS, Horike S, Itakura T, et al. Enhanced and optically switchable proton conductivity in a melting coordination polymer crystal. Angew Chem Int Ed, 2017, 56: 4976-4981

20 Yin L, Huang W, Xiao R, et al. Optically stimulated synaptic devices based on the hybrid structure of silicon nanomembrane and perovskite. Nano Lett, 2020, 20: 3378-3387

$21 \mathrm{Xu}$ XY, Yan B. Intelligent molecular searcher from logic computing network based on $\mathrm{Eu}(\mathrm{III})$ functionalized UMOFs for environmental monitoring. Adv Funct Mater, 2017, 27: 1700247

22 Yu K, Wei T, Li Z, et al. Construction of molecular sensing and logic systems based on site-occupying effect-modulated MOF-DNA interaction. J Am Chem Soc, 2020, 142: 21267-21271

23 Zhang Y, Yan B. MIL-61 and Eu ${ }^{3+} @$ MIL-61 as signal transducers to construct an intelligent boolean logical library based on visualized luminescent metal-organic frameworks. ACS Appl Mater Interfaces, 2019, 11: 20125-20133

24 Singh A, Verma P, Laha S, et al. Photochromic conjugated microporous polymer manifesting bio-inspired pcFRET and logic gate functioning. ACS Appl Mater Interfaces, 2020, 12: 20991-20997

25 Xiao J, Liu J, Liu M, et al. Fabrication of a luminescence-silent system based on a post-synthetic modification Cd-MOFs: A highly selective and sensitive turn-on luminescent probe for ascorbic acid detection. Inorg Chem, 2019, 58: 6167-6174

26 Xiong X, Xiao M, Lai W, et al. Optochemical control of DNA-switching circuits for logic and probabilistic computation. Angew Chem Int Ed, 2020, 60: 3397-3401

27 Fan S, Wang S, Wang X, et al. Photogated proton conductivity of ZIF-8 membranes co-modified with graphene quantum dots and polystyrene sulfonate. Sci China Mater, 2021, 64: 1997-2007

28 Liang HQ, Guo Y, Shi Y, et al. A light-responsive metal-organic framework hybrid membrane with high on/off photoswitchable proton conductivity. Angew Chem Int Ed, 2020, 59: 7732-7737

29 Wen Y, Sheng T, Zhu X, et al. Introduction of red-green-blue fluorescent dyes into a metal-organic framework for tunable white light emission. Adv Mater, 2017, 29: 1700778

30 Wang X, Li Z, Ying W, et al. Blue metal-organic framework encapsulated denatured R-phycoerythrin proteins for a white-light-emitting thin film. J Mater Chem C, 2020, 8: 240-246

31 Wen Y, Liu Q, Su S, et al. Coordination tailoring of water-labile 3D MOFs to fabricate ultrathin 2D MOF nanosheets. Nanoscale, 2020, 12: $12767-12772$

32 Desmettre T, Devoisselle JM, Mordon S. Fluorescence properties and metabolic features of indocyanine green (ICG) as related to angiography. Surv Ophthalmol, 2000, 45: 15-27

33 Fang $\mathrm{C}$, Yan $\mathrm{P}$, Ren $\mathrm{Z}$, et al. Multifunctional $\mathrm{MoO}_{2}$-ICG nanoplatform for $808 \mathrm{~nm}$-mediated synergetic photodynamic/photothermal therapy. Appl Mater Today, 2019, 15: 472-481

34 Lim DW, Kitagawa H. Rational strategies for proton-conductive metalorganic frameworks. Chem Soc Rev, 2021, 50: 6349-6368

35 Rice AM, Martin CR, Galitskiy VA, et al. Photophysics modulation in photoswitchable metal-organic frameworks. Chem Rev, 2020, 120: 8790-8813

36 Wang Z, Cohen SM. Postsynthetic modification of metal-organic frameworks. Chem Soc Rev, 2009, 38: 1315-1329

37 Jiang $\mathrm{K}$, Zhang L, Hu Q, et al. Indocyanine green-encapsulated nanoscale metal-organic frameworks for highly effective chemo-photothermal combination cancer therapy. Mater Today Nano, 2018, 2: 5057

38 Wang T, Li S, Zou Z, et al. A zeolitic imidazolate framework-8-based indocyanine green theranostic agent for infrared fluorescence imaging and photothermal therapy. J Mater Chem B, 2018, 6: 3914-3921

$39 \mathrm{Wu} \mathrm{B}, \mathrm{Fu}$ J, Zhou Y, et al. Metal-organic framework-based chemophotothermal combinational system for precise, rapid, and efficient antibacterial therapeutics. Pharmaceutics, 2019, 11: 463

40 Wang $\mathrm{H}, \mathrm{Li} \mathrm{X}$, Tse BWC, et al. Indocyanine green-incorporating nanoparticles for cancer theranostics. Theranostics, 2018, 8: 1227-1242

41 Jiang X, Du B, Huang Y, et al. Cancer photothermal therapy with ICGconjugated gold nanoclusters. Bioconjug Chem, 2020, 31: 1522-1528

42 Mao Y, Li J, Cao W, et al. General incorporation of diverse components inside metal-organic framework thin films at room temperature. Nat Commun, 2014, 5: 5532

43 Gholibegloo E, Karbasi A, Pourhajibagher M, et al. Carnosine-graphene oxide conjugates decorated with hydroxyapatite as promising nanocarrier for ICG loading with enhanced antibacterial effects in photodynamic therapy against streptococcus mutans. J Photochem Photobiol B-Biol, 2018, 181: 14-22

44 Zhang R, Ji S, Wang N, et al. Coordination-driven in situ self-assembly strategy for the preparation of metal-organic framework hybrid membranes. Angew Chem Int Ed, 2014, 53: 9775-9779

45 Yang C, Xu J, Yang D, et al. ICG@ZIF-8: One-step encapsulation of indocyanine green in ZIF-8 and use as a therapeutic nanoplatform. Chin Chem Lett, 2018, 29: 1421-1424

46 Guo Y, Jiang Z, Ying W, et al. A DNA-threaded ZIF-8 membrane with high proton conductivity and low methanol permeability. Adv Mater, 2017, 30: 1705155

47 Kreuer KD, Rabenau A, Weppner W. Vehicle mechanism, a new model for the interpretation of the conductivity of fast proton conductors. Angew Chem Int Ed, 1982, 21: 208-209

48 Agmon N. The Grotthuss mechanism. Chem Phys Lett, 1995, 244: 456462

49 Liu C, Yang D, Jin Q, et al. A chiroptical logic circuit based on selfassembled soft materials containing amphiphilic spiropyran. Adv Mater, 2016, 28: 1644-1649

50 Kim C, Favazza C, Wang LV. In vivo photoacoustic tomography of chemicals: High-resolution functional and molecular optical imaging at new depths. Chem Rev, 2010, 110: 2756-2782

51 de la Zerda A, Bodapati S, Teed R, et al. Family of enhanced photoacoustic imaging agents for high-sensitivity and multiplexing studies in living mice. ACS Nano, 2012, 6: 4694-4701

$52 \mathrm{Yu}$ J, Javier D, Yaseen MA, et al. Self-assembly synthesis, tumor cell targeting, and photothermal capabilities of antibody-coated indocyanine green nanocapsules. J Am Chem Soc, 2010, 132: 1929-1938

53 Zheng X, Xing D, Zhou F, et al. Indocyanine green-containing nanostructure as near infrared dual-functional targeting probes for optical imaging and photothermal therapy. Mol Pharm, 2011, 8: 447-456

54 Li L, Dong X, Li J, et al. A short review on NIR-II organic small molecule dyes. Dyes Pigm, 2020, 183: 108756

Acknowledgements This work was supported by the National Natural Science Foundations of China (NSFC 21875212), the Key Program of National Natural Science and Foundation (NSFC 51632008), Major R \& D plan of Zhejiang Natural Science Foundation (LD18E020001), and the Fundamental Research Funds for the Central Universities.

Author contributions Peng X supervised the project; Fan S performed the experiments, analyzed the results, and wrote the manuscript; Wang $\mathrm{X}$, Wan $\mathrm{X}$, and Fang $\mathrm{Z}$ assisted in the device fabrication and measurement; Pi $\mathrm{X}$ and $\mathrm{Ye} \mathrm{Z}$ contributed to the theoretical and data analysis. All authors contributed to the general discussion.

Conflict of interest The authors declare that they have no conflict of interest.

Supplementary information Supporting data are available in the online version of the paper. 


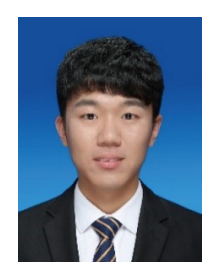

Shuaikang Fan received his bachelor degree (2019) from the College of Materials Science and Engineering at Sichuan University. He is currently pursuing his Master degree at the School of Materials Science and Engineering, Zhejiang University under the supervision of Prof. Xinsheng Peng. His present research interest mainly focuses on the design and synthesis of proton conductive MOF membranes for energy conversion devices.

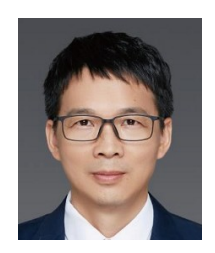

Xinsheng Peng received his $\mathrm{PhD}$ degree in 2003 at the Institute of Solid State Physics, Chinese Academy of Sciences. He became a full professor at the School of Materials Science and Engineering, Zhejiang University in 2010. His research interest mainly focuses on the design and synthesis of functional membranes and controlled mass transportation in energy and environmental science.

\section{光控质子逻辑门: 吲哚菁绿修饰的HSB-W5纳米片薄膜}

范帅康 ${ }^{1}$, 王世林 ${ }^{1}$, 王小涁 ${ }^{1}$, 万昕艺 ${ }^{1}$, 方舟 $^{1}$, 皮孝东 ${ }^{1}$, 叶志镇 $^{1,2}$, 彭新生 $1,2^{*}$

摘要 光控质子传导在先进传感器和信息处理设备中有重要的应用前 景. 本文通过将吗哚菁绿(ICG)引入HSB-W5基体中, 制备出一种利用光 热转换实现光控质子传导性能的金属有机骨架 (MOFs) 薄膜 (ICG@HSB-W5), 并以此为基础构建了NOT、NAND和NOR逻辑门. ICG@HSB-W5 薄膜的质子电导率是HSB-W5薄膜的 25 倍; 而且该薄膜 的质子电导率对激光具有高效快速的响应, 开关比超过 1000 , 可以形成 不同的电导状态来构建逻辑门. 在这个逻辑门系统中, 808-和 $405-\mathrm{nm}$ 的 激光作为输入信号, 质子电导率作为输出信号. 质子电导率作为一种电 信号可根据实际需求调节阈值, 也能方便地被接收和检测. 本文为 MOF 基分子逻辑门的设计提供了一种新的途径并有助于质子逻辑门甚至更 复杂的逻辑器件的发展. 